\title{
Introduction to the special issue on IEEE-NEWCAS 2012
}

\author{
Patricia Desgreys - Massimo Alioto
}

Published online: 26 September 2013

(c) Springer Science+Business Media New York 2013

This special issue of Analog Integrated Circuits and Signal Processing (ALOG) includes the extended version of a limited number of papers that were originally presented at the IEEE International NEWCAS 2012 conference, held in Montreal (Canada) on June 18-21, 2012. Since its birth, the NEWCAS conference has consistently grown to become a premiere venue where highly innovative circuit and system techniques have been presented for the first time. The conference covers a wide variety of subjects including analog and RF circuit design, digital and mixed-signal integrated circuits, computer architecture, signal processing, sensors, encompassing a broad range of applications such as telecommunications, imaging and vision, safety, energy and biomedical.

In the 2012 NEWCAS edition, the submissions reached the record number of 314 from 43 countries from all over the world. After a rigorous review process, 138 contributions were accepted for presentation. The $43 \%$ acceptance rate is the lowest ever recorded at NEWCAS. This accomplishment fits well the general trend of high and yet increasing quality of the conference, which has been inspired this year by the vision of the NEWCAS2012 General Chairs, Prof. Mounir Boukadoum and Prof. Mohamad Sawan.

Among the contributions published in the NEWCAS2012 proceedings, 22 papers were selected and invited

\footnotetext{
P. Desgreys $(\square)$

Communications and Electronics Department, Telecom

ParisTech, Paris, France

e-mail: patricia.desgreys@telecom-paristech.fr
}

\section{Alioto}

Department of Electrical and Computer Engineering, National University of Singapore, Singapore, Singapore

e-mail: massimo.alioto@gmail.com for submission to this special issue. After the review process, 14 papers have been accepted for publication on this special issue. In this selection, many other fine contributions would have reserved publication on this issue, but the limited number of pages did not allow it.

The contributions included in this special issue address various challenges that are within the scope of NEWCAS, as listed below:

(1) Analog and RF circuit design: temperature compensated CMOS voltage-controlled oscillator for gas sensor, interactive CMOS circuit sizing, tunable stopband active filter, multi-path inductorless TIAs for larger transimpedance limit.

(2) Digital and mixed signals: residue-weighted number conversion, reconstruction scheme for M-channel TI ADCs, high-level synthesis of multi-stage noise band cancellation $\Sigma \Delta$ ADC.

(3) Low-power: ultra-low power microsystem for hydrogen gas sensing, low-power FPGA based asynchronous wake-up receiver, power-sensitivity trade-off in TRT receivers.

(4) Heterogeneous modeling: HW-SW fully WSN simulation and UML metamodel, compact modeling of vertical Hall-effect devices.

(5) Sensors: microwave-based biosensor,

(6) Signal processing: oversampled multi-phase timedomain BER processing.

We would like to thank the authors for their scientific contributions, and the numerous reviewers for their constructive evaluations. We really enjoyed interacting with them, they really made this journey exciting. We would also like to express our appreciation for the continuous and tireless support of Prof. Mohamed Ismail, as well as our gratitude to the Springer personnel for their support. 
In the end, this special issue was made possible by the personal contribution of many people. We take the opportunity offered by this Editorial to thank each and all of them.

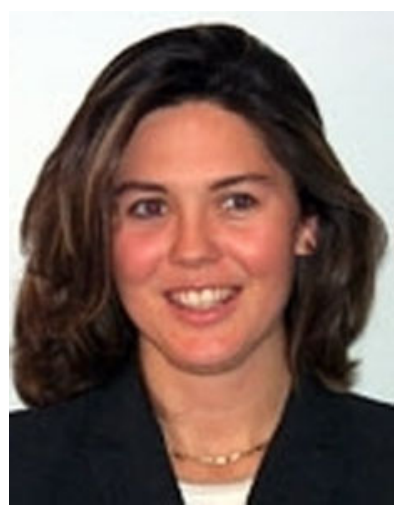

Patricia Desgreys (M'00SM'12) received the Ph.D. degree in Microelectronics in 1999 from the University of Bordeaux I, France. In 2010, she received the HdR degree (authorization to manage research) in Engineering Sciences from the University of Paris VI, France. Since 2000, she is associate professor in the Circuit and Communication Systems group, at the Communications and Electronics Department of Institute MinesTelecom-TELECOM ParisTech and she is heading this group since 2013. She is in charge of research on Wireless Systems and Design Techniques for Nanoscale Circuits with special emphasis on Software and Cognitive Radio System. So far, she has co-authored more than 80 technical publications in international reviews and conference proceedings and has been involved in many collaborative projects. Patricia Desgreys is a board member of the LTCI (Processing and Information Communication Laboratory), CNRS research laboratory of Telecom ParisTech. She is also animator of its thematic network within the Mines-Telecom Institute. Since 2007, she is a member of the Steering Committee of CNRS Research group on SoC-SiP in charge of animating the community of 600 French researchers on $\mathrm{SoC}-\mathrm{SiP}$ design. In this context, she has organized more than ten workshops on hot topics with international renowned speakers. Patricia is an IEEE Senior Member, involved in the international animation of the CAS community in particular Technical Program Chair of IEEE NEWCAS for 2012 and 2013 and Guest Editor of special issues in Analog Integrated Circuits and Signal Processing.

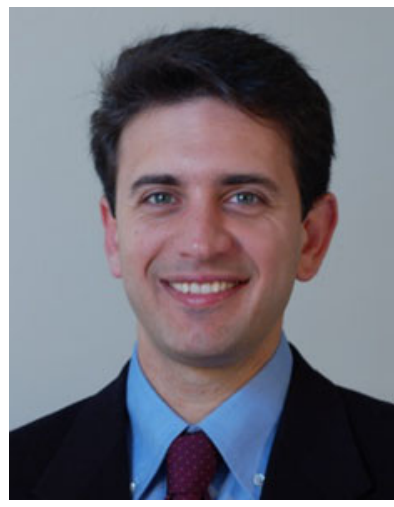

Massimo Alioto (M'01SM'07) was born in Brescia, Italy, in 1972. He received the Laurea (M.Sc.) degree in Electronics Engineering and the Ph.D. degree in Electrical Engineering from the University of Catania (Italy) in 1997 and 2001, respectively. He is an Associate Professor at the Department of Electrical and Computer Engineering, National University of Singapore. Previously, he was Associate Professor at the Department of Information Engineering of the University of Siena. In
2013 he was Visiting Scientist at Intel Labs-CRL (Oregon) to work on ultra-scalable microarchitectures. In 2011-2012, he was Visiting Professor at University of Michigan, Ann Arbor, investigating on active techniques for resiliency in near-threshold processors, erroraware VLSI design for wide energy scalability, self-powered circuits. In 2009-2011, he was Visiting Professor at BWRC-University of California, Berkeley, investigating on next-generation ultra-low power circuits and wireless nodes. In the summer of 2007, he was a Visiting Professor at EPFL-Lausanne (Switzerland). He has authored or co-authored more than 180 publications on journals $(65+$, mostly IEEE Transactions) and conference proceedings. Two of them are among the most downloaded TVLSI papers in 2007 (respectively 10 th and 13th). He is co-author of two books, Flip-Flop Design in Nanometer CMOS-from High Speed to Low Energy (Springer, 2013) and Model and Design of Bipolar and MOS Current-Mode Logic: CML, ECL and SCL Digital Circuits (Springer, 2005). His primary research interests include ultra-low power VLSI circuits, selfpowered and wireless nodes, near-threshold circuits for green computing, error-aware and widely energy-scalable VLSI circuits, circuit techniques for emerging technologies. Prof. Alioto is an IEEE Senior Member, and was a member of the HiPEAC Network of Excellence (EU) and the MuSyC FCRP Center (US). In 2010-2012 he was the Chair of the "VLSI Systems and Applications" Technical Committee of the IEEE Circuits and Systems Society, for which he was also Distinguished Lecturer in 2009-2010 and member of the DLP Coordinating Committee in 2011-2012. He currently serves as Associate Editor in Chief of the IEEE Transactions on VLSI Systems, and served as Guest Editor of various journal special issues (including the issue on "Ultra-Low Voltage Circuits and Systems for Green Computing" published on December 2012 on IEEE Transactions on Circuits and Systems-part II). He also serves or has served as Associate Editor of a number of journals (IEEE Transactions on VLSI Systems, ACM Transactions on Design Automation of Electronic Systems, IEEE Transactions on CAS-part I, Microelectronics Journal, Integration-The VLSI journal, Journal of Circuits, Systems, and Computers, Journal of Low Power Electronics, Journal of Low Power Electronics and Applications). He was Technical Program Chair of the ICECS 2013, NEWCAS 2012 and ICM 2010 conferences, and Track Chair in a number of conferences (ICCD, ISCAS, ICECS, VLSI-SoC, APCCAS, ICM). 